\title{
Field joint coating technology for horizontal directional drilling of oil and gas pipeline
}

\author{
Jie Ding ${ }^{1, \mathrm{a}}$, Ting Lei ${ }^{2}$, Ansheng Zheng ${ }^{3}$ and Jianjun $\mathrm{Li}^{1}$ \\ ${ }^{1}$ Engineering, Corrosion \& Protection, China Petroleum Pipeline Engineering Corporation, China \\ ${ }^{2}$ Engineering, Water and Wastewater, China Petroleum Pipeline Engineering Corporation, China \\ ${ }^{3}$ Senior Engineering, Corrosion \& Protection, China Petroleum Pipeline Engineering Corporation, China
}

\begin{abstract}
Horizontal Directional Drilling (HDD) technology is an important way crossing a river for pipeline. However, due to the impact of different geological conditions, construction technology and other factors, usually, pipeline surface coating is damaged during HDD pullback process. Especially field joint coating position has been the weakest section of the HDD integrity protection. This article describes the main damage form of coating for HDD pipeline, as well as performance, features and applications of a variety of field joint coatings used for HDD pipeline, and the prospect for HDD pipeline integrity protection.
\end{abstract}

Keywords: horizontal directional drilling; field joint coating; pipeline; corrosion protection.

\section{Introduction}

Pipeline HDD technology was originated from oilfield drilling technology. During the early 1970s, the United States began using horizontal directional drilling technology. The technology uses drilling rig installed on the ground. And drill bit is driven by the drill pipe to drill into the ground and form a pilot hole by a smaller angle relative to the ground. The reamer is then driven by the drilling rig and the diameter of drive hole is extended for the laying of pipeline. Finally pullback the pipeline by drilling rig and complete the construction [1]. Since HDD has many advantages, such as not damaging the surface environment, short construction period, relatively low cost and so on, it has been an important way crossing a river for pipeline.

However, due to the impact of different geological conditions, construction technology and other factors, usually, pipeline surface coating is damaged during HDD pullback process, worse the pipe body might be scratched, which greatly affects the coating integrity and the service life of pipeline itself. The HDD pipeline field joint coating is the weakest part of entire pipeline integrity protection. Field joint coating material and the construction process determines the coating quality, thereby affecting the health of the entire pipeline. Therefore, the selection of suitable coating materials and construction process is particularly important for HDD pipeline crossing construction process.

\section{Surveys}

In recent years, usually 3PE coating is used for HDD pipeline anti-corrosion and field joint coating

${ }^{a}$ Corresponding author : cppedingjie @e-mail.org 
adopts HDD special heat shrinkable tape in China. The related statistics was taken for HDD pipeline coating damage form, the details as shown as follows, and the field joint coating damage is also a major factor.

1) Axial scratch coating, as shown in Figure 1;

2) Circumferential scratch coating, as shown in Figure 2;

3) Heat shrink tape off, as shown in Figure 3;

4) Curl at lap joint between heat shrink tape and main coating, as shown in Figure 4;

5) Coating sag, as shown in Figure 5;

6) Other.
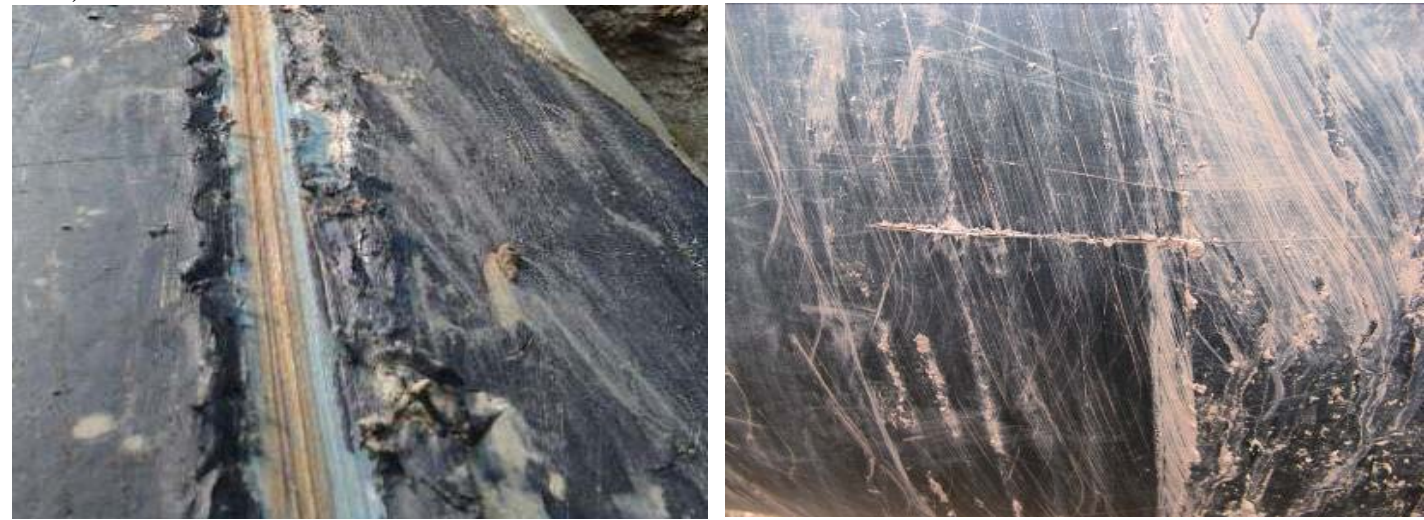

Figure 1. Axial scratch coating

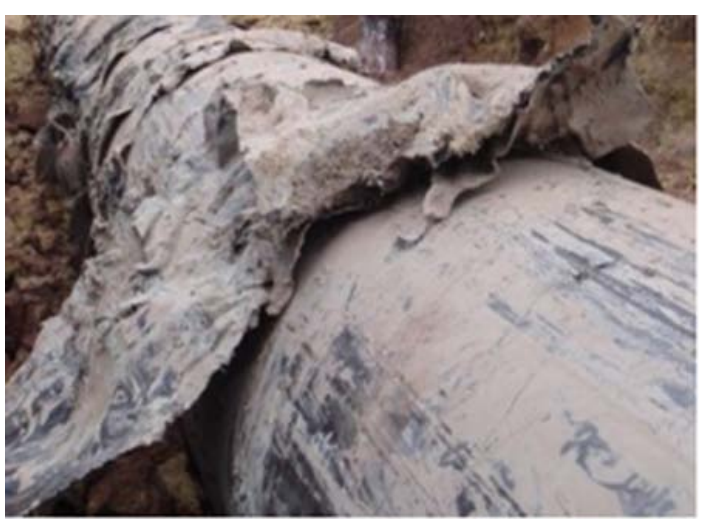

Figure 3. Heat shrink tape off
Figure 2. Circumferential scratch coat

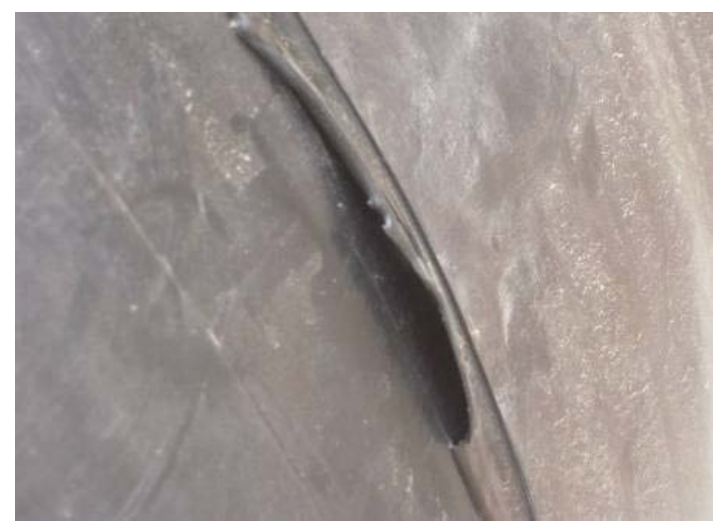

Figure 4. Curl at lap joint

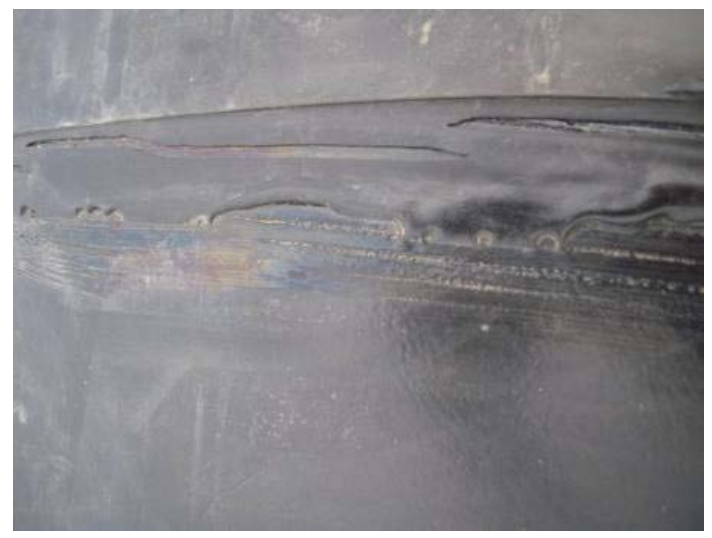

Figure 5. Coating sag 


\section{Field joint coating technology}

\subsection{Solvent-free epoxy primer + Heat shrinkable tape}

\subsubsection{Solvent-free epoxy primer}

Solvent-free epoxy primer is also known as high-strength epoxy paint. The main film-forming material is bisphenol-A modified epoxy resin, reactive toughening diluted polyurethane as curing agent to achieve free solvent. Due to the high solids reactive epoxy, curing film cycle is short, no solvent evaporation, a film of up to $200 \mu \mathrm{m}$ or more, high solid content, high hardness, strength, no pinhole defects. Solvent-free epoxy primer has excellent insulation property, excellent chemical stability as solvent epoxy coating, and the adhesion, toughness, impact strength, corrosion resistance, peel strength has been greatly improved. Especially in terms of corrosion resistance, it has good acid resistance, oil resistance, alkali resistance, salt spray corrosion resistance and other chemical characteristics. It also has excellent wear resistance, stamping resistance, scrub resistance and other physical characteristics. The construction process is simple, saving energy, no environmental pollution. It is mainly used in urban pipeline corrosion, buried pipeline internal and external corrosion, field joint coating, bend, buried pipeline coating repair and other heavy-corrosion areas.

\subsubsection{Heat shrinkable tape}

Heat shrinkable tape is designed and manufactured for buried and overhead steel pipe anti-corrosion and insulation outer surface. And it is mainly used for pipe bending parts and pipeline damaged coating repair, it can also be used for entire pipe enwinding and field joint coating. It is a composite of radiation cross-linked polyolefin substrates and special sealed hot melt. The special sealed hot melt can form a good bond among polyethylene substrate, steel surfaces and solid epoxy coating. When heating and installing a heat shrinkable tape, substrate will be shrunk in radial direction, while the internal composite adhesive layer melts, and wraps the joint tightly. The adhesive layer forms a solid anti-corrosion shell together with the substrate on the pipeline body, which can resist soil stress, anti-hydrostatic pressure, resistance to cathodic disbonding, chemical anti-corrosion and bacteria corrosion. Heat shrinkable tape has excellent wear resistance, corrosion resistance, impact resistance and good resistance to UV and photo-aging properties [2, 3].

\subsubsection{Application}

Solvent-free epoxy primer + Heat shrinkable tape for HDD, this field joint coating can be applied for 3PE coating pipeline. During construction process, the surface treatment and preheating shall be carried out, and then paint solvent-free epoxy primer. After the thickness and curing has met the requirements of the related codes, install heat shrinkable tape and sacrificial tape special for HDD at HDD pullback direction. 24 hours after the completion of construction, the relevant testing shall be carried out to ensure construction quality.

The advantages of the field joint coating is that the adhesive of heat shrinkable tape contains modified EVA or butyl rubber, which are non-polar and has good compatibility with non- polar PE material. The adhesive contains tackifying resin, which has good adhesive force with bare pipe and solvent-free epoxy primer, so the pipe is firmly bonded together with the field joint coating when the heat shrinkable tape is shrunk at radial direction, which plays a part in seal and anti-corrosion. During the pullback process of HDD, the installation of sacrificial tape special for HDD can effectively reduce the damage to heat shrinkable tape, while the heat shrinkable tape can form a better protection to solvent-free epoxy primer. Since the construction is convenient and cost-effective, the field joint coating of heat shrinkable tape has become a common field joint coating way of 3LPE HDD pipeline in service. Many HDD pipeline adopt this field joint coating way, such as China West-East Gas 
Pipeline Project, Lanzhou - Chengdu crude oil pipeline project. But through the feed detection of many as-built HDD pipelines crossing quality in recent years, it was found that when the HDD pipeline cross the rock area or the related construction technology is unsuitable, the field joint coating also has been damaged during pullback process, which affects anti-corrosion quality and service life of the pipeline. So in such cases, a more reasonable and effective field joint coating way for HDD shall be considered.

\subsection{Solvent-free epoxy coating + Solvent-free epoxy abrasion resistant coating}

\subsubsection{Solvent-free epoxy abrasion resistant coating}

The composition and performance of solvent-free epoxy coating has been introduced in section 3.1. Solvent-free epoxy abrasion resistant coating uses epoxy resin as the base material, adding high wear resistant filler and corresponding assistant, modified aliphatic amine as curing agent, to obtain a better modified solvent-free epoxy coating, which has higher scratch resistance, abrasion resistance, impact resistance, hardness and better adhesion.

\subsubsection{Application}

Solvent-free epoxy coating + Solvent-free epoxy abrasion resistant coating, this field joint coating can be applied for the pipeline which is coated by double fusion bonded epoxy powder. During construction process, solvent-free epoxy coating shall be mixed and painted uniformly in accordance with product requirements. The coating thickness shall be controlled to ensure the quality of the coating, and the coating dry film thickness shall not be less than $800 \mu \mathrm{m}$. After the solvent-free epoxy coating is surface dry, paint solvent-free epoxy abrasion resistant coating, and the coating film total thickness shall not be less than $1.6 \mathrm{~mm}$. The width of lap between field joint coating and main pipeline coating shall not be less than $100 \mathrm{~mm}$, and the coating shall be gradually thinning and has a smooth transition at the overlapping area. The coating surfaces shall be smooth, seamless, sagging and no other appearance defects.

The advantage of the field joint coating is that the field construction is convenient. And the field joint coating has well anti soil stress and mechanical properties, chemical resistance and cathodic disbonding resistance. The epoxy coating has micro-absorption of water, which can make micro-current of cathodic protection passing and does not form cathodic protection shield. Epoxy coatings have good adhesion. The base material of under layer solvent-free epoxy coating and surface layer abrasion resistant coating is the same, so the two coatings can be fully compatible with each other. The adhesion strength between under layer epoxy coating and steel is more than 10MPa.The adhesive strength between under layer epoxy coating and fusion bonded epoxy powder coating is more than $7 \mathrm{MPa}$, so that the paint is firmly bonded together with the steel body surface, and can also be compatible with the pipeline coating to form a unified entire external coating. During HDD pullback process, the field joint coating cannot disbond from the steel body, and will not become the weak link of the overall corrosion coating. The excellent properties of surface layer solvent-free epoxy abrasion resistant coating can effectively prevent damage of the coating by obstructions in the drill bore, to ensure the integrity of the corrosion coating. As for certain harsh geological conditions, the entire HDD pipeline can be painted by solvent-free epoxy abrasion resistant coating to improve scratch resistance, wear resistance, hardness and other properties, in order to achieve more effective protection. Domestic Hohhot-Baotou-Erdos Product Oil Pipeline Project Yellow River HDD uses the method, which is coated by solvent-free epoxy abrasion resistant entirely. The coating (The under layer: Pipeline coating is double fusion bonded epoxy powder coating and the field joint coating is solvent-free epoxy coating) achieve a better crossing protection. 


\subsection{Solvent-free epoxy / Heat shrinkable tape+ Epoxy glass fiber reinforced plastic}

\subsubsection{Epoxy glass fiber reinforced plastic}

Epoxy glass fiber reinforced plastics (Epoxy FRP) uses epoxy resin as adhesives, adding curing agents, powder etc to achieve adhesive materials, and uses glass fiber or its products as reinforced materials, to gain a low-pressure molding composite materials through the two interface recombination by a certain molding process.

1) Epoxy resin

Epoxy resin is defined as organic macromolecular compounds whose molecules have two or more epoxy groups. The molecular structure of the epoxy resin is that molecular chain contains reactive epoxy groups, and the epoxy group is at the end or middle of the molecular chain or as a cyclic structure. Since the molecular structure contains the reactive epoxy group, they can cross link and react with various types of curing agent to form insoluble, infusible and three-to mesh structure polymer.

Cured epoxy resin has good physical and chemical properties. It has excellent adhesion strength to the surface of metallic and nonmetallic materials, good dielectric properties, good dimensional stability, high hardness, good flexibility, anti-corrosion properties, which is widely used in various sectors of national defense and economy, for pouring, dipping, laminating materials, adhesives, coatings and other uses. As anti-corrosion material, epoxy resin not only has dense, water-resistant, anti-leakage, high strength characteristics, but also has strong adhesion, room temperature operation, simple construction and other good properties. Also the cost is affordable. China studied the epoxy resin since 1958, and put into industrial production quickly. Until now it has been booming in the country. In addition to the production of ordinary bisphenol, A-epichlorohydrin type epoxy resin, various types of new epoxy resin is also produced, in order to meet the urgent need of national defense and national economic sectors [4].

2) Glass fiber cloth

Glass fiber cloth is woven by inorganic glass fibers. And its characteristics such as weight, thickness, fracture strength and other properties are determined by fiber character, the density, the weave structure. Glass fiber cloth has alkali-resistant, acid-resistant, water-resistant, anti-corrosion, fire resistance, insulation, high strength, impact resistance and good adhesion with resin. It mainly used as insulation material, fiberglass reinforced material, chemical filter cloth, high-pressure steam heat insulation material, fire protection product, high elastic belt, building material and wall covering.

Glass fiber cloth used for HDD is generally alkali-free, non-twist, wax-free glass fiber roving plain fabric, which is important substrate for manual glass fiber reinforced plastic. During glass fiber cloth making process, since the glass is brittle, the thick glass fiber is easy to break. So the diameter of fiber used for roving plain fabric is generally $12 \sim 23 \mu \mathrm{m}$. Because more parallel strands or roving comprised by parallel monofilament is arranged at warp and weft direction for the glass fiber cloth, the strength of the glass fiber cloth at warp and weft direction is greater.

\subsubsection{Application}

Solvent-free epoxy primer/Heat shrinkable tape+ Epoxy FRP, this field joint coating can be applied for the 3PE-coating pipeline. During construction process, firstly complete the installation of the solvent-free epoxy primer and heat shrinkable tape, then inspect, if the inspection is acceptable, epoxy FRP is coated. And the structure of epoxy FRP is generally "two cloth five resin", ie paint epoxy resin (surface dry) + epoxy resin+ Glass fiber cloth+ epoxy resin+ Glass fiber cloth + epoxy resin (surface dry) + epoxy resin, the number of glass fiber cloth layer and the thickness of epoxy FRP can be increased properly according to the project actual needs, such as "three cloth six resin", "four cloth seven resin".

The main features of epoxy FRP is excellent corrosion resistance, shear resistance and tensile resistance, hardness, compressive strength, impact resistance, scratch resistance, abrasion resistance, 
as well as excellent adhesion to other materials, integrity, and it also can effectively isolate the various media and have high resistance to cathodic disbanding. It can protect the anti-corrosion coating adequately when HDD pipeline crosses the adverse geological area, such as hard rock area. Epoxy FRP is applied for armoring coating of heat shrinkable tape, which is used for HDD crossing. The advantages of this field joint coating way is easy to construct at field. As armoring coating, epoxy FRP improve compressive strength, scratch resistance and abrasion resistance properties greatly for the field joint coating. And because epoxy FRP has excellent adhesion to PE material, it is firmly bonded together with heat shrinkable tape to prevent disbonding due to the adhesion is not enough. Epoxy FRP can form an effective protection for HDD field joint coating. As for more demanding HDD crossing, such as the crossing area is hard bedrock, gravel, sandstone and so on, because the geological conditions is complex , the formed hole is unsatisfactory mostly after reaming, and the mud ratio is irrational, the pipeline coating is easy to damage during pullback process. In order to achieve more effective protection, the entire HDD pipeline can be painted by epoxy FRP. The thickness can be determined according to the actual situation. Some domestic River HDD crossing, such as Lanzhou Zhengzhou - Changsha Product Oil Pipeline Project Changjiang River and West-East pipeline Jiangyin Changjiang River, use the method which is coated by epoxy FRP entirely ( the under layer: Pipeline coating is 3PE and the field joint coating is heat shrinkable tape ) and achieve excellent cross protection.

\subsection{Solvent-free polyurethane coating}

\subsubsection{Solvent free polyurethane coating}

Polyurethane is a new organic polymer material and is known as "fifth largest plastic". Because of its superior performance, it is widely used in many areas of the national economy, such as light industry, chemical industry, electronics, building materials, automotive, defense and so on.

Polyurethane coating uses polyurethane resin as the main film material. Polyurethane resin is a polymeric compound, which is generally obtained by reaction between isocyanate compound and active hydrogen compound (such as hydroxyl group, a carboxyl group, etc.). Its molecular structure has repeating urethane link, so called polyurethane resin. Solvent-free polyurethane coating is obtained by the reaction between polyisocyanate solution and polybasic alcohol solution. The reaction forms polyurethane layer at required surface. Under normal circumstances, the coating is in liquid state, but turn into $100 \%$ solid coating after painting. The coating does not contain organic volatile substances, harmful substances (such as amine, coal tar, isocyanate monomer) and combustible substances. The molecular structure of the coating film is a highly cross linked network structure, which has outstanding performance, such as adhesion resistance, excellent chemical resistance, good resistance to impact and abrasion, excellent flexibility, resistance to cathodic disbonding, fast curing, environment adaptability and long service life[5,6].

\subsubsection{Application}

Solvent-free polyurethane coatings can be applied for the 3PE-coating pipeline. During construction process, it can be sprayed, brushed and roller coated, etc. The coating dry film thickness shall be not less than 2mm, and the width of lap between field joint coating and main pipeline coating shall be not less than $100 \mathrm{~mm}$. When using high-pressure airless spray, set the mixing proportion, preheat, insulate for the coating in accordance with the instruction requirements to ensure good atomization. As for brush, roller coating way, a person shall be designated for the preparation and mixing of coating in strict accordance with product instructions, and use the coating in the applicable period. Coating dwell should be avoided to prevent reducing wetting to the substrate due to increased viscosity.

Using solvent-free polyurethane coatings as field joint coating of 3PE coating HDD pipeline, the coating has excellent wear and corrosion resistance as well as prominent adhesion towards pipe body and PE coating. And it can form an effective protection for field joint, which combines dual 
performance of the anti-corrosion and armoring coating. The coating at overlapping area has a smooth transition. Pipeline coating is thicker than the field joint coating, which prevents the formation of "hump" at HDD pullback direction and can effectively prevent damage of the coating by obstructions in the drill bore. The field joint coating cannot disbond from the pipe body and achieve a good protective effect.

\subsection{Solvent free polyurethane coating+ Epoxy glass fiber reinforced plastic.}

For some particularly harsh geological conditions, HDD pipeline coating if using 3PE, solvent-free polyurethane coating can be used as field joint coating. And after qualified examination of solvent-free polyurethane, adopt epoxy FRP to carry out continuous coating for HDD entire pipeline, ie, the field joint coating is solvent free polyurethane coating+ epoxy FRP. This approach combines the advantages of two excellent coating. The under layer solvent-free polyurethane coating not only has good corrosion resistance, prominent adhesion towards steel body and PE, but also has wear resistance, impact resistance, scratch resistance and other properties like armoring coating. After coating epoxy FRP, a solid protection for pipeline coating and field joint coating is formed. And the formed overall coating has further improvement at wear resistance, scratch resistance, hardness, etc. The epoxy FRP armoring coating, 3PE anti-corrosion pipeline coating and solvent free polyurethane field joint coating form an organic whole. This method can achieve excellent protection for HDD pipeline.

\section{Conclusion and prospect}

As for 3PE-coating HDD pipeline, if the geological condition is kindly and the drill hole is satisfied, solvent-free epoxy primer + heat shrinkable tape or solvent-free polyurethane coating can be used for field joint coating; if the geological condition is demanding, complex and strictly, the drill hole is not satisfied, solvent-free epoxy / heat shrinkable tape+ epoxy glass fiber reinforced plastic or solvent free polyurethane coating+ Epoxy glass fiber reinforced plastic can be used for field joint coating, and if more excellent cross protection want to be achieved, the entire HDD pipeline armoring coating shall be considered.

With the social, scientific and technological progress, diameter of construction pipeline is also growing, and the requirements of the anti-corrosion coating and armoring coating for HDD pipeline are higher. So based on optimization of HDD technology, researching, developing and applying better anti-corrosion and armoring coating and field joint coating is necessary, and the coating shall be more suitable for HDD technology and geological conditions. During the applying process, HDD process conditions, technical feasibility, cost-effective and environmentally friendly and other factors must be taken into account. Study the corrosion mechanism at breakage section of HDD pipeline and improve damage resistance and anti-corrosion resistance of the coating. Research the construction technology, such as ratio of mud, crossing curve, site painting and coating process. Improve the coating performance evaluation and related support systems after HDD crossing, to ensure the service life of HDD pipeline. And there is great practical significance and economic benefits for HDD pipeline protection.

\section{References}

1. Zhao S. Horizontal Directional Drilling for Oil and Gas Pipeline [J]. Petroleum Engineering Construction, 35(2): 37-40 (2009).

2. Hu S.X., Liao Y.P. and Wang B.H. Handbook of pipeline coating designing. (2007).

3. Chen H. Optimization of field joint coating for buried steel pipeline [J]. Pipeline Technology and Equipment, 1:39-42 (2005).

4. Liu X. Anti-corrosion coating and painting application. (2008). 
5. Meng F.Q., Yao H.J. and Sun H.L. Solvent-free polyurethane coating applications in large-diameter steel pipeline boring project [J]. China Water \& Wastewater, 23(2): 96-98 (2007).

6. Niu L.M. and Li, J. Application of new material for pipeline corrosion- $100 \%$ solids polyurethane anti-corrosion coating [J].Oil Knowledge, 1: 24-26(2004). 